\title{
Summer-flowering species of maculate Aloe L. (Asphodelaceae: Alooideae) in the Aloe zebrina-complex from South Africa: reinstatement of four names, and description of $A$. braamvanwykii Gideon F.Sm. \& Figueiredo
}

\author{
Gideon F. Smith ${ }^{1,2,3}$, Estrela Figueiredo ${ }^{3,4}$, Ronell R. Klopper ${ }^{1,2}$ and Neil R. Crouch ${ }^{5,6}$ \\ ${ }^{1}$ Biosystematics Research and Biodiversity Collections Division, South African National Biodiversity Institute, \\ Private Bag X101, Pretoria, 0001 South Africa (email: G.Smith@sanbi.org.za; R.Klopper@ sanbi.org.za). \\ ${ }^{2}$ H.G.W.J. Schweickerdt Herbarium, Department of Plant Science, University of Pretoria, Pretoria, 0002 \\ South Africa. \\ ${ }^{3}$ Centre for Functional Ecology, Departamento de Ciências da Vida, Universidade de Coimbra, 3001-455 \\ Coimbra, Portugal. \\ ${ }^{4}$ Department of Botany, P.O. Box 77000, Nelson Mandela Metropolitan University, Port Elizabeth, 6031 \\ South Africa (email: estrelafigueiredo@ hotmail.com). \\ ${ }^{5}$ Ethnobotany Unit, South African National Biodiversity Institute, P.O. Box 52099, 4007 Berea Road, \\ South Africa (email: N.Crouch@sanbi.org). \\ ${ }^{6}$ School of Chemistry and Physics, University of KwaZulu-Natal, 4041 Durban, South Africa.
}

Summary: A new restricted range species of maculate Aloe L. (Asphodelaceae: Alooideae), A. braamvanwykii Gideon F.Sm. \& Figueiredo is described from near Wolmaransstad in the NorthWest Province of South Africa. The species is related to five other summer-flowering maculate aloes in the A. zebrina-complex: A. ammophila Reynolds, A. komatiensis Reynolds, A. lettyae Reynolds, $A$. transvaalensis Kuntze (probably its nearest relative) and $A$. vandermerwei Reynolds, but differs from these five species in several aspects. Aloe ammophila, A. komatiensis, A. transvaalensis and $A$. vandermerwei have previously been included in the synonymy of $A$. zebrina Baker, and are here reinstated. Aloe braamvanwykii is adapted to the climatic extremes of the country's central north-western grasslands where it is subject to very low winter and high summer temperatures.

Zusammenfassung: Es wird eine neue geflecktblättrige Art von Aloe L. (Asphodelaceae: Alooideae), A. braamvanwykii Gideon F.Sm. \& Figueiredo, aus der Nähe von Wolmaransstad in der North-West-Provinz von Südafrika beschrieben. Die Art ist mit fünf weiteren sommerblühenden, gefleckt-blättrigen Arten aus $\operatorname{dem} A$. zebrina-Komplex verwandt: $A$. ammophila Reynolds, A. komatiensis Reynolds, A. lettyae Reynolds, A. transvaalensis Kuntze (möglicherweise die am nächsten verwandte Art) und $A$. vandermerwei Reynolds, unterscheidet sich aber in verschiedenen Merkmalen von diesen fünf Arten. Aloe ammophila, A. komatiensis, A. transvaalensis and $A$. vandermerwei wurden bisher als Synonyme zu A. zebrina Baker gestellt, werden hier aber wieder als eigenständige Arten betrachtet. Aloe braamvanwykii ist an die klimatischen Extreme der zentral-nordwestlichen Grasländer Südafrikas angepasst, wo sie sehr tiefen Winterund hohen Sommertemperaturen ausgesetzt ist.

\section{Introduction}

Taxonomically, the summer-flowering maculate aloes (Aloe L.; Asphodelaceae: Alooideae) from central southern Africa resolve as follows: $A$. $z e b$ rina Baker is a predominantly near-tropical element that stretches across south-tropical Africa (essentially the Flora zambesiaca area) from the Angolan coast to central Mozambique; A. ammophila Reynolds is in large part a north-central element from around, and north of, Polokwane (Pietersburg) in the Limpopo Province of South Africa; A. komatiensis Reynolds is an eastern element occurring from Malelane and Komatipoort in Mpumalanga, South Africa, to Maputo in Mozambique; A. lettyae Reynolds also occurs to the east in the Duiwelskloof and Magoebaskloof areas of the Limpopo Province, South Africa; A. transvaalensis Kuntze is most frequent around 


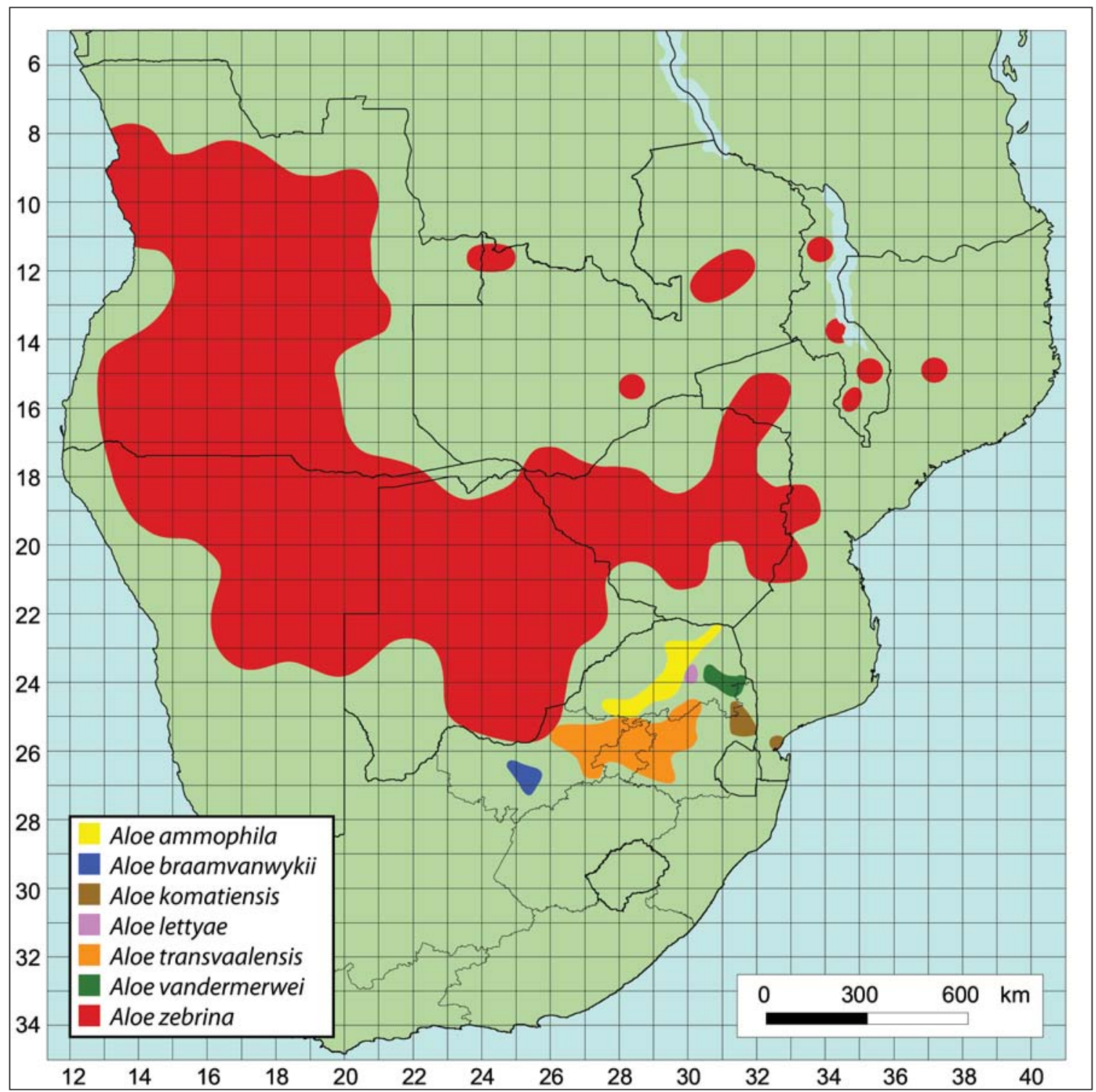

Figure 1. Distribution of members of the A. zebrina-complex in southern Africa.

South Africa's central Gauteng Province, in the vicinity of Johannesburg and Pretoria, and $A$. vandermerwei is again a more eastern element from the Gravelotte, Letaba and Timbavati region in the Limpopo Province, South Africa (Figure 1). Material from the southern parts of the North-West Province of South Africa, however, is not accommodated within the concepts of any of these species. We therefore here describe Aloe braamvanwykii Gideon F.Sm. \& Figueiredo as new.

Recent literature has treated A. zebrina as a very widespread and variable species, by including into its synonymy A. ammophila,
A. transvaalensis and A. vandermerwei (Van Wyk \& Smith, 1996; Glen \& Hardy, 2000; Carter et al., 2011) and sometimes also A. lettyae (Glen \& Hardy, 2000). The latter species has, however, been retained by many authors as a good species (Van Wyk \& Smith, 1996; Carter et al., 2011), a view with which we agree. We believe that $A$. ammophila, A. transvaalensis and A. vandermerwei as treated by Reynolds (1950), differ sufficiently from $A$. zebrina to warrant recognition as separate species, and therefore reinstate these names here.

A further species falling within this group of summer-flowering maculate aloes, is A. komatiensis. The affinities of this species are not as 


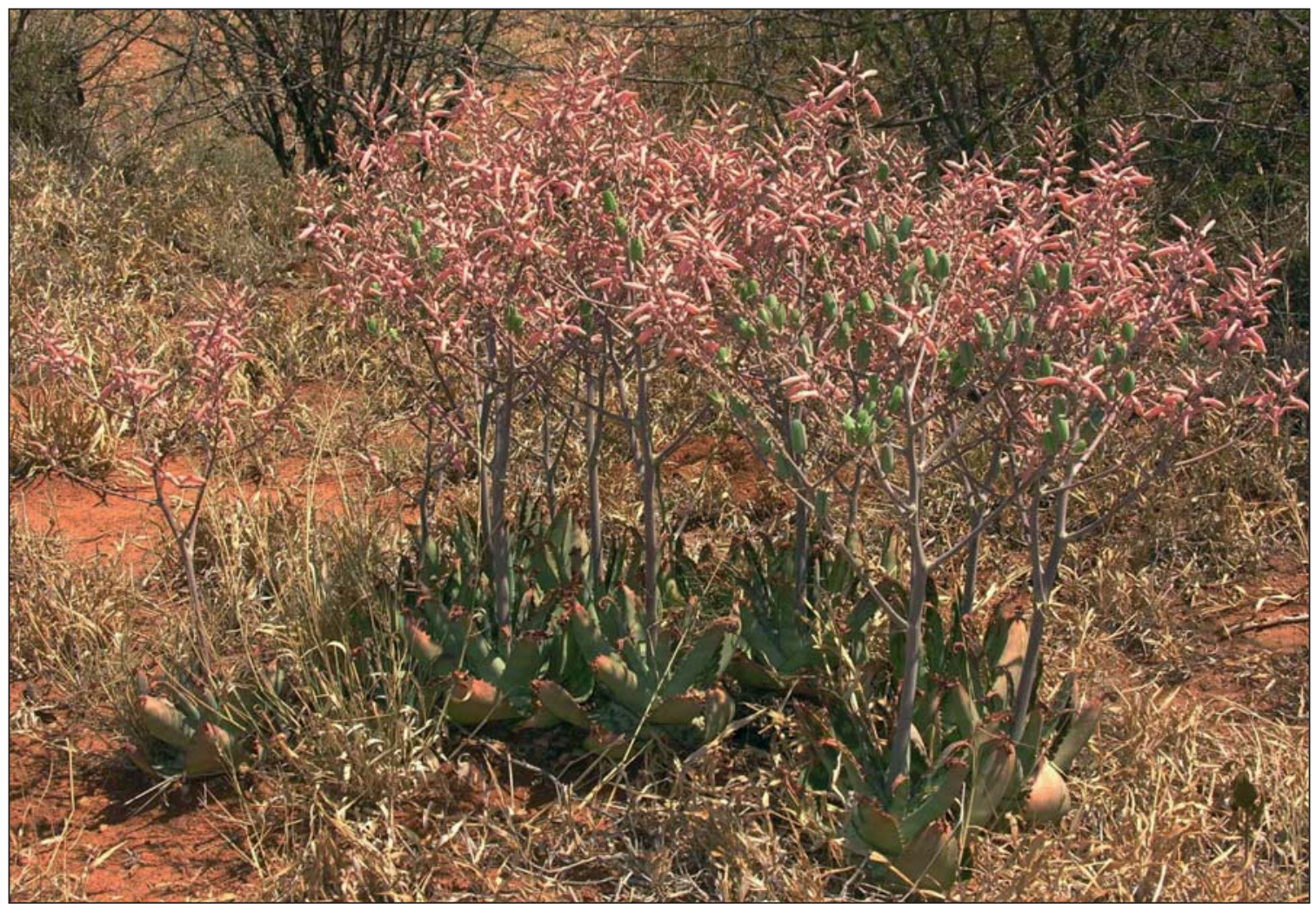

Figure 2. A. ammophila forms large dense colonies. Photo: Philip Nel.

clear-cut: it has been variously included in the synonymy of A. zebrina (Glen \& Hardy, 2000) and A. parvibracteata Schönland (Van Wyk \& Smith, 1996; Carter et al., 2011). Both A. lusitanica Groenew. and $A$. decurvidens Groenew. were regarded by Reynolds (1950) as conspecific with A. komatiensis. Subsequently, this broadened concept for A. komatiensis was synonymised under A. parvibracteata by Van Wyk \& Smith (1996). In contrast, Glen \& Hardy (2000) placed $A$. lusitanica under A. parvibracteata, but synonymised Reynolds' 1936 concept of $A$. komatiensis under A. zebrina. Glen \& Hardy (2000) did not treat $A$. decurvidens, although in a preceding account (Glen et al., 1995) $A$. decurvidens was considered a synonym of $A$. zebrina, with $A$. lusitanica conspecific with $A$. parvibracteata (as a component of the synonymous $A$. komatiensis). Aloe parvibracteata flowers in winter (June-July), unlike $A$. komatiensis, $A$. lusitanica and $A$. decurvidens which are all recorded as flowering in summer (between February and April) (Groenewald, 1937a \& b; Reynolds, 1950). We believe that $A$. komatiensis represents a further element of the summer-flowering maculate aloe complex that warrants recognition as a separate taxon and reinstate this name here.
Diagnostic characters and information on the distribution and habitat for each of the summer-flowering maculate aloes in the A. zebrinacomplex within South Africa is provided. Differences among the species are presented in Table 1.

\section{Species treatments}

Aloe ammophila Reynolds in J. S. Afr. Bot. 2: 116 (1936). Type: South Africa, Limpopo Province, Pietersburg, on road to Chuniespoort, flowered in Johannesburg 14 March 1936, G.W. Reynolds 1345 (PRE, holo.!)

Diagnostic characters: This aloe typically forms large dense colonies of up to 100 plants. Leaves are usually clearly spotted in transverse bands on the lower surface. Inflorescences are up to $0.66 \mathrm{~m}$ high and widely branched from about the middle with long, wide and divaricate branches so that the inflorescence is often wider than it is high (Figure 2). Flowers are $30-33 \mathrm{~mm}$ long and coralred, with a $1 \mathrm{~mm}$ wide white border on the outer perianth segments.

Distribution: It is centred around Polokwane (Pietersburg), but occurs from the Bela-Bela (Warmbad) and Mookgophong (Naboomspruit) 


\begin{tabular}{|c|c|c|c|c|c|c|}
\hline 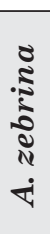 & 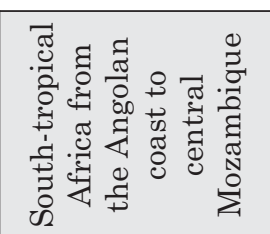 & 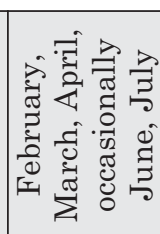 & 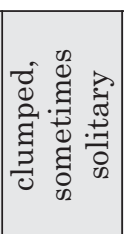 & $\begin{array}{l}\varepsilon \\
0 \\
0 \\
0 \\
\times \\
0 \\
0 \\
1 \\
0 \\
0\end{array}$ & 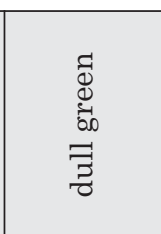 & 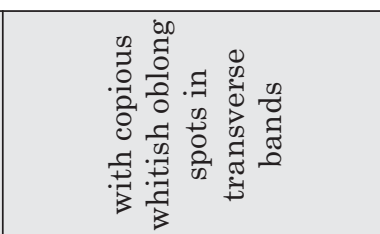 \\
\hline 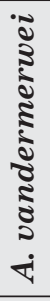 & 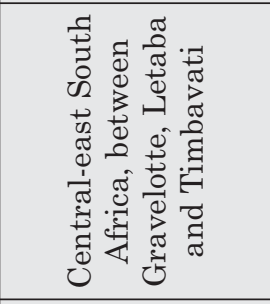 & 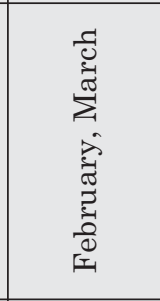 & 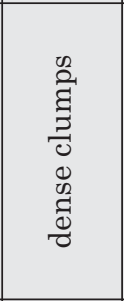 & 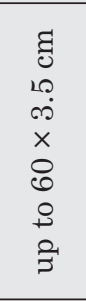 & 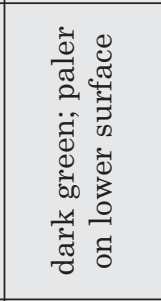 & 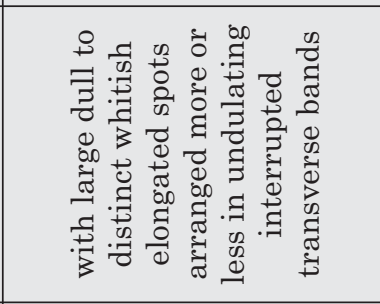 \\
\hline 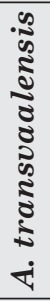 & 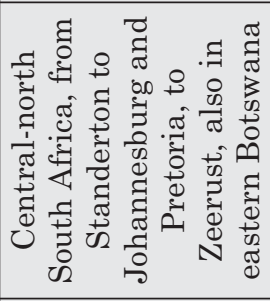 & 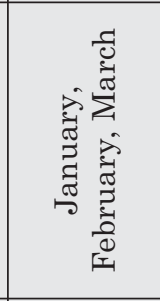 & 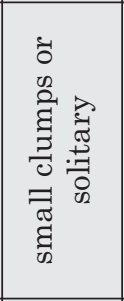 & 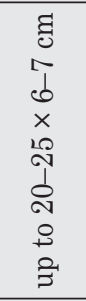 & 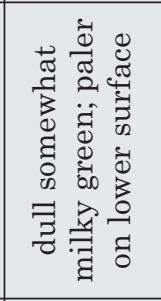 & 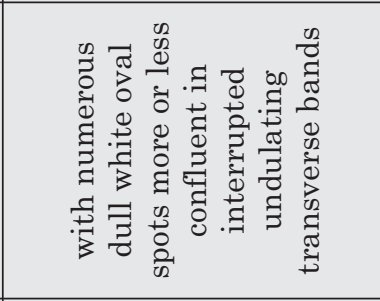 \\
\hline 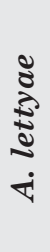 & 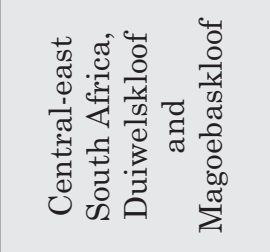 & 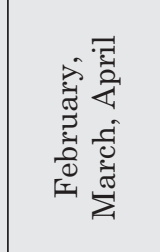 & 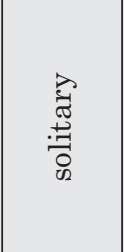 & $\begin{array}{l}\Xi \\
0 \\
0 \\
\times \\
10 \\
+1 \\
0 \\
+ \\
\vdots\end{array}$ & 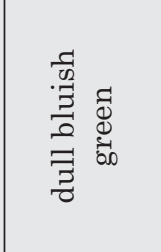 & 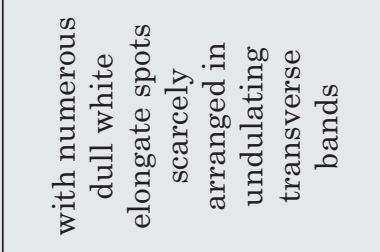 \\
\hline 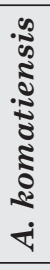 & 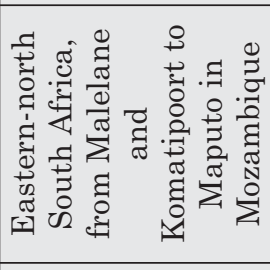 & 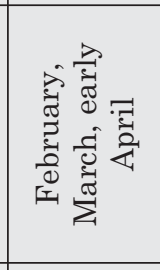 & 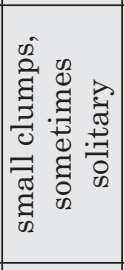 & $\begin{array}{l}q \\
0 \\
\circ \\
+ \\
\infty \\
\infty \\
\times \\
0 \\
+ \\
+1\end{array}$ & 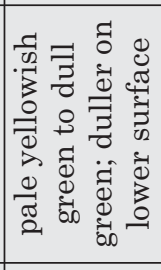 & 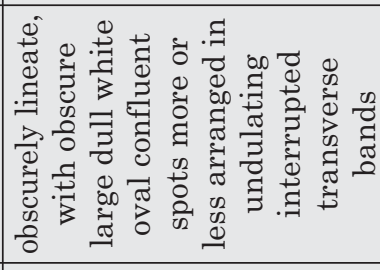 \\
\hline 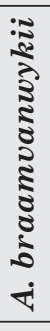 & 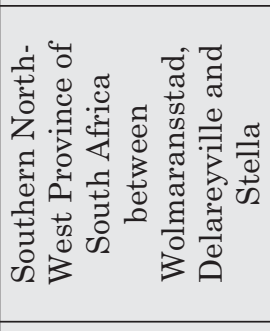 & 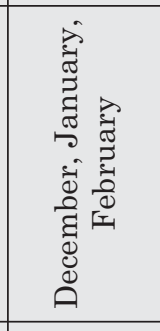 & 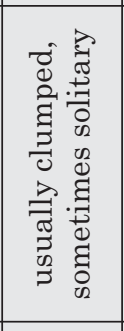 & 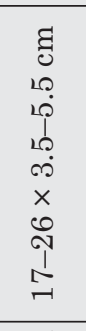 & 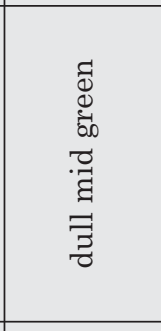 & 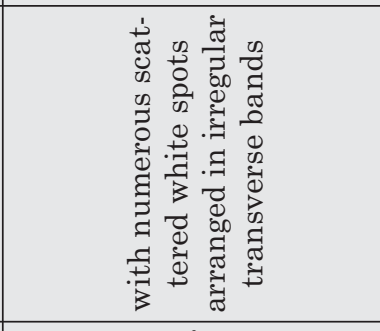 \\
\hline 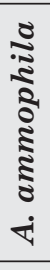 & 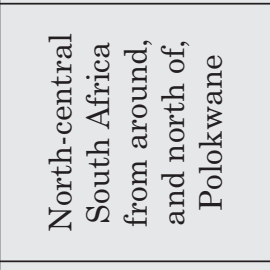 & 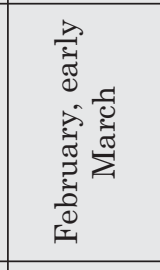 & 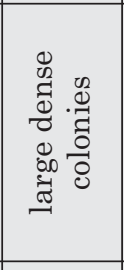 & 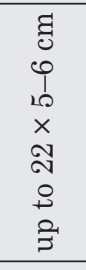 & 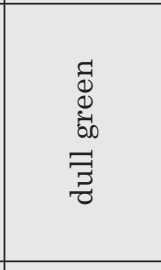 & 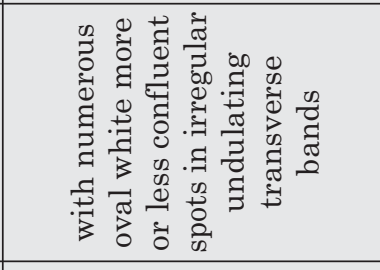 \\
\hline 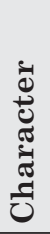 & 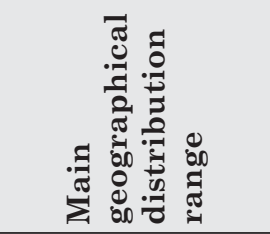 & 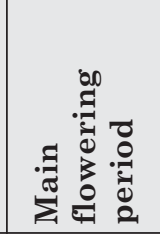 & 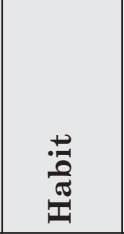 & 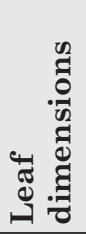 & 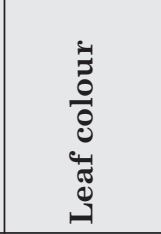 & 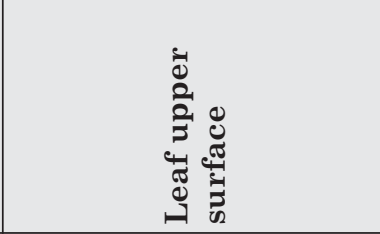 \\
\hline
\end{tabular}




\begin{tabular}{|c|c|c|c|c|c|c|c|}
\hline 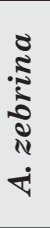 & 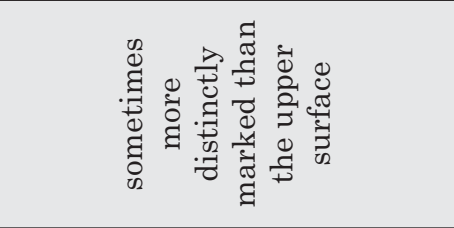 & $\begin{array}{l}q \\
0 \\
0 \\
-1 \\
0 \\
-\end{array}$ & 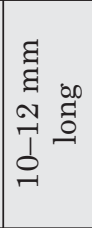 & 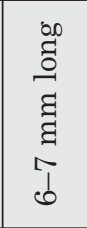 & 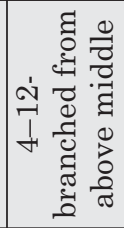 & 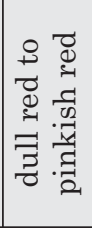 & 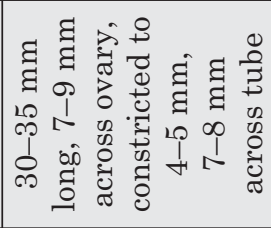 \\
\hline 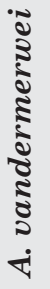 & 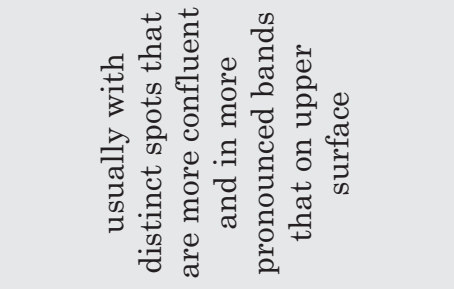 & $\begin{array}{l}\text { q } \\
\text { ने } \\
+\end{array}$ & 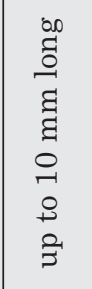 & 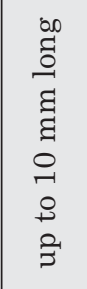 & 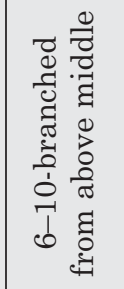 & 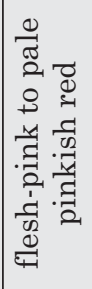 & 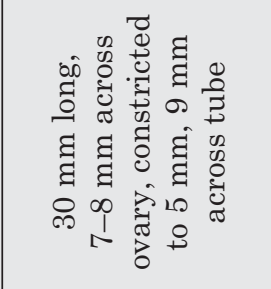 \\
\hline 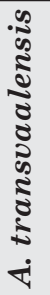 & 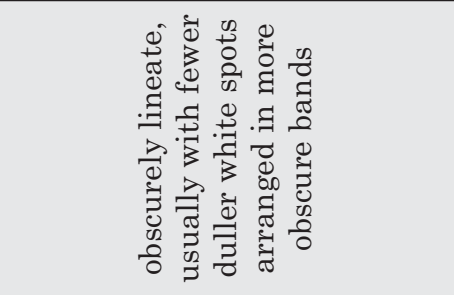 & $\begin{array}{l}\varepsilon \\
10 \\
10 \\
\\
0 \\
-\end{array}$ & 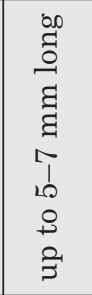 & 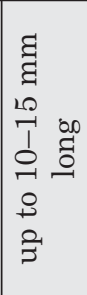 & 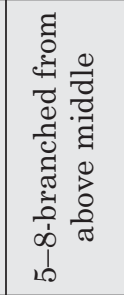 & 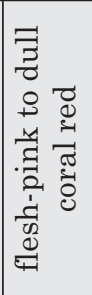 & 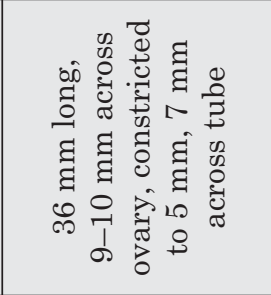 \\
\hline 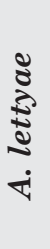 & 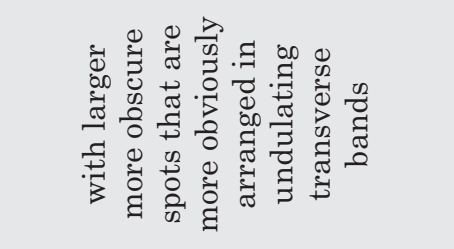 & 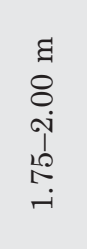 & 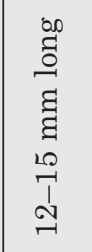 & 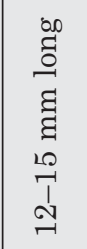 & 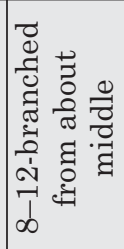 & 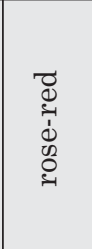 & 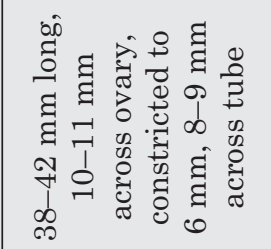 \\
\hline 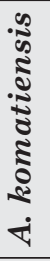 & 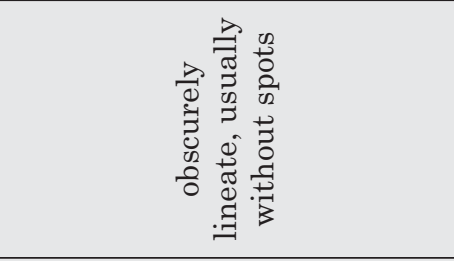 & 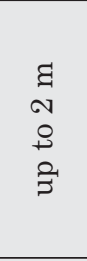 & 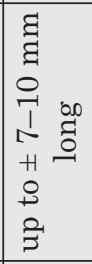 & 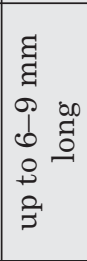 & 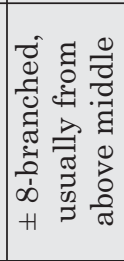 & 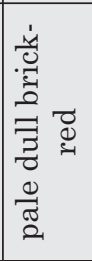 & 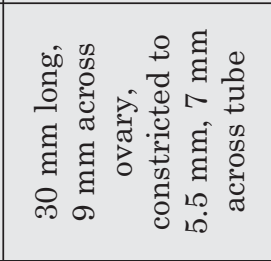 \\
\hline 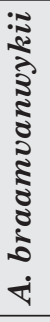 & 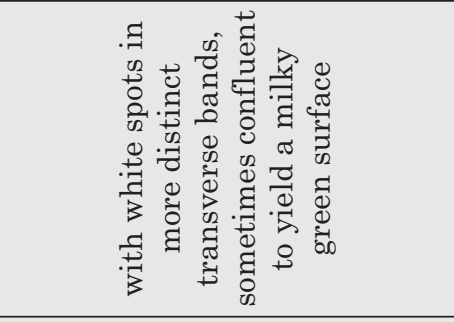 & $\begin{array}{l}q \\
10 \\
10 \\
0 \\
10 \\
0 \\
0 \\
0\end{array}$ & $\begin{array}{l}\infty \\
0 \\
0 \\
\text { घี } \\
\text { घี } \\
0 \\
1 \\
10\end{array}$ & 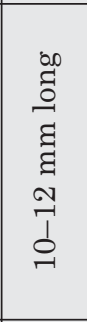 & 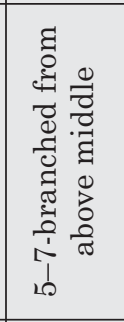 & 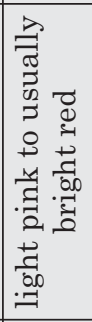 & 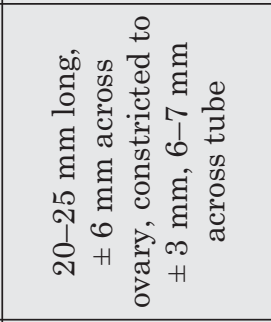 \\
\hline 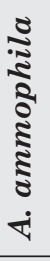 & 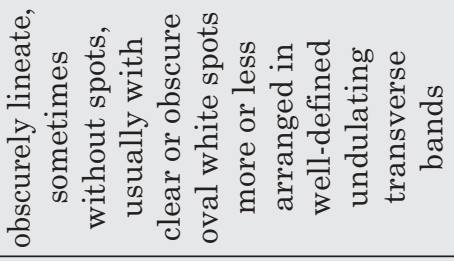 & $\begin{array}{l}\Xi \\
\vdots \\
\vdots \\
0 \\
0 \\
H\end{array}$ & 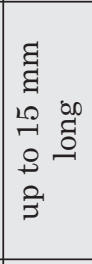 & 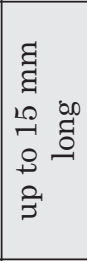 & 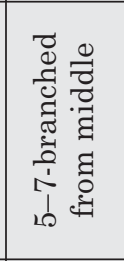 & 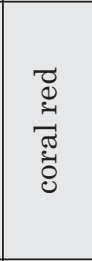 & 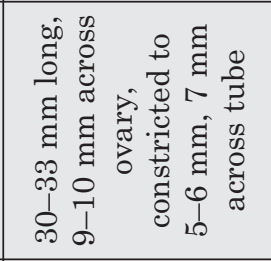 \\
\hline 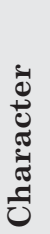 & 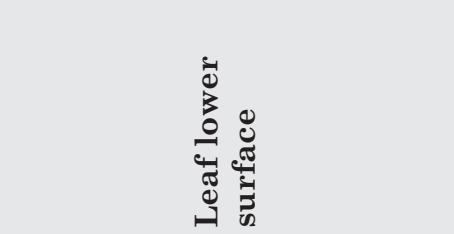 & 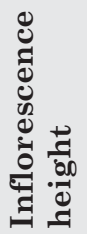 & 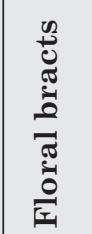 & $\begin{array}{l}\frac{\pi}{2} \\
0 \\
0 \\
0 \\
0 \\
0\end{array}$ & 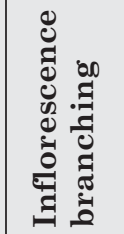 & 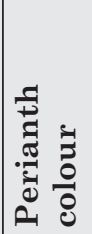 & 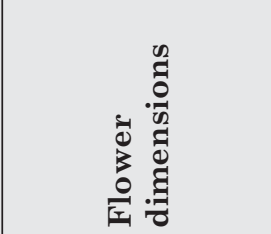 \\
\hline
\end{tabular}


area, northwards to Louis Trichardt, Wylies Poort and Musina, Limpopo Province, South Africa.

Habitat: Flat places, in rich sandy soil, usually in grassveld, sometimes in clearings among acacia and other shrubs.

Aloe komatiensis Reynolds in J. S. Afr. Bot. 2: 120 (1936). Type: South Africa, Mpumalanga, Komatipoort, flowered in Johannesburg 24 March 1936, G.W. Reynolds 1543 (PRE, holo.!; BOL, iso.!)

A. decurvidens Groenew. in Tydskr. Wetensk. Kuns 15: 126 (1937). Type: South Africa, Pietersburg district, F.Z. van der Merwe 107 (PRE, holo.!)

A. lusitanica Groenew. in Tydskr. Wetensk. Kuns 16: 13 (1937). Type: Mozambique, Maputo, April 1937, F.Z. van der Merwe s.n. in PRE24087 (PRE, lecto.!) (designated by Glen et al. 1995: 98)

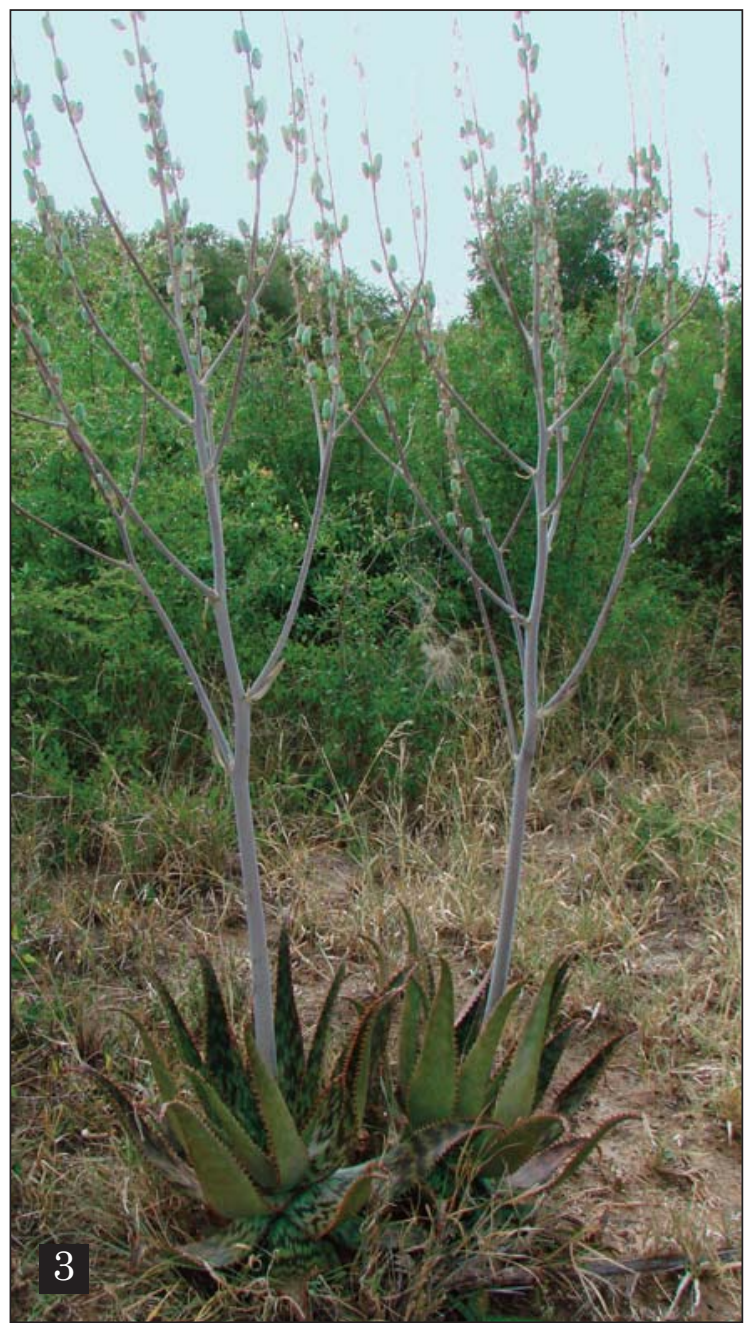

Diagnostic characters: This aloe sometimes occurs as solitary rosettes, but usually forms small groups. Leaves are very narrow, yellowish green to dull green and usually without spots on the lower surface. Peduncle and flowers are lightly covered with a grey powdery bloom. Inflorescences are up to $2 \mathrm{~m}$ high and compactly branched from above the middle (Figure 3). Flowers are $\pm 30 \mathrm{~mm}$ long and pale dull brick red, with a $1 \mathrm{~mm}$ wide dull white border on the outer perianth segments.

Distribution: It occurs from Komatipoort southwards to Figtree and westwards to Malelane, Mpumalanga, South Africa, and also in Mozambique between Maputo and Marracuene.

Habitat: Grassveld and bushveld in a summer rainfall area that is free from frost.

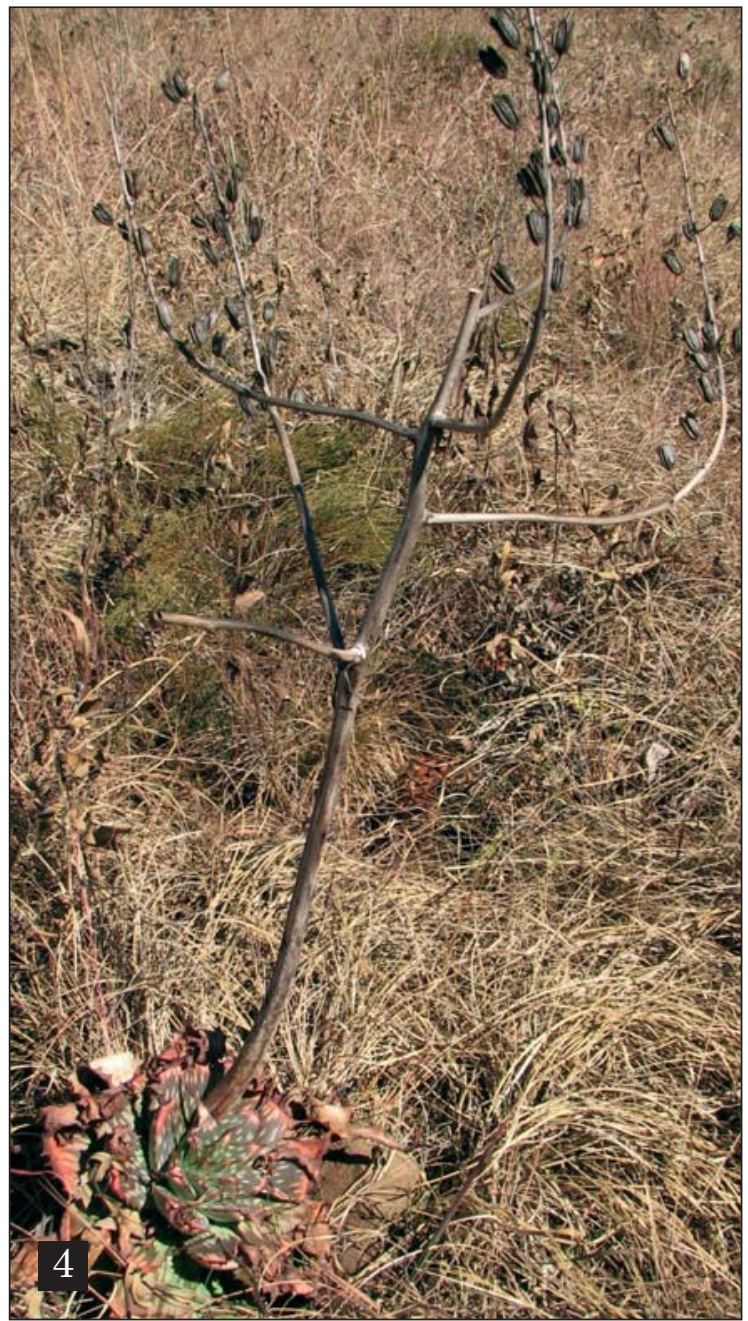

Figure 3. A. komatiensis has tall inflorescences of up to $2 \mathrm{~m}$, and leaves that are usually unspotted on the under surface. Photo: Willem Froneman. Figure 4. A. lettyae in fruit, showing the characteristic orientation of the inflorescence branches. Photo: Erich van Wyk. 
Aloe lettyae Reynolds in J. S. Afr. Bot. 3: 137 (1937). Type: South Africa, Limpopo Province, Duiwelskloof, 26 March 1937, G.W. Reynolds 2339 (PRE, holo.!; BOL, iso.!)

Diagnostic characters: This aloe occurs as solitary plants. Leaves are obscurely spotted on the lower surface. Inflorescences are 1.75-2.00 $\mathrm{m}$ high and branched from about the middle with the branches distinctly rounded and gracefully curved (Figure 4). Flowers are 38-42 $\mathrm{mm}$ long and rosered, with pale margins and a remarkably large globose base.

Distribution: It is only known from the area around Duiwelskloof and Magoebaskloof, Limpopo, South Africa.

Habitat: Long grass and among bushes and trees on eastern slopes of hills and mountains.

Aloe transvaalensis Kuntze in Revisio Generum Plantarum 3(2): 314 (1898). Type: South Africa, Gauteng, Pretoria, 17 February 1894, Kuntze s.n. (NY, holo.; K, iso.!)

A. laxissima Reynolds in J. S. Afr. Bot. 2: 28 (1936). Type: South Africa, Limpopo Province, near Nebo, March 1935, G.W. Reynolds 767 (PRE, holo.!)

Diagnostic characters: This aloe occurs as solitary plants or in small groups, only occasionally in large groups. Leaves are usually a dull milky green, with the lower surface paler green and more obscurely spotted in less defined bands than the upper surface, to unspotted. Inflorescences are $\pm 1 \mathrm{~m}$ high and compactly branched from above the middle (Figure 5). Flowers are $\pm 36 \mathrm{~mm}$ long and flesh-pink with a $1 \mathrm{~mm}$ wide white border on the outer perianth segments.

Distribution: It is centred in central South Africa, especially in the Gauteng province around Pretoria and Johannesburg, also southeast towards Heidelberg and to Standerton in Mpumalanga, and westwards to Rustenburg and Zeerust in the northern parts of the North-West province. It is also reported from near Serowe and Mabela-ePudi in Botswana (see for example Hargreaves, 1990), but the identity of material from these localities requires confirmation.

Habitat: Rocky slopes, often at the foot of koppies, frequently between shrubs and bushes.

Aloe vandermerwei Reynolds in The aloes of South Africa: 268 (1950). A. angustifolia Groenew. (nom. illegit.) in Flow. Pl. S. Afr. 18: t.708 (1938). Type: South Africa, Limpopo Province, near Gravelotte, April 1936, F.Z. van der Merwe s.n. in PRE21288 (PRE, holo.)
Diagnostic characters: This aloe occurs in dense groups. Leaves are remarkably long (up to $60 \mathrm{~cm}$ ) relative to their width $(3.5 \mathrm{~cm})$, spreading and downwards curved, often twisted and intermingled giving it a snake-like appearance (Figure 7). Leaves are usually distinctly spotted on the lower surface, with spots more confluent and in more pronounced bands than the upper surface. Inflorescences are up to $1 \mathrm{~m}$ high and branched from above the middle. Flowers are $30 \mathrm{~mm}$ long and flesh-pink, with a $1.0-1.5 \mathrm{~mm}$ wide whitish border on the outer perianth segments.

Distribution: It is occurs from between Leydsdorp and Gravelotte, westwards to Malopene and Letaba in the Kruger National Park, and southwards to the Timbavati area, Limpopo, South Africa.

Habitat: Red clayey soil in grassveld and lowveld in openings among acacia and other trees.

Aloe zebrina Baker in Trans. Linn. Soc. London, Bot. 1: 264 (1878). Type: Angola, Loanda District, Barra do Bengo, Quicuxe towards Cacuaco, July 1854, F. Welwitsch 3721 (LISU, lecto.; BM!, G!, K!, isolecto.)

A. bamangwatensis Schönland in Rec. Albany Mus. 1: 122 (1904). Type: Botswana, Palapye Road, March 1904, S. Schönland 1656 (GRAA7223) (GRA, holo.; PRE, iso.!)

A. baumii Engl. \& Gilg in O.Warburg (ed.), Kunene-Sambesi Expedition: 191 (1903). Type: Angola, Chirumbu, 14 October 1899, H. Baum 275 (B, holo.; E, iso.)

A. constricta Baker in J. Linn. Soc., Bot. 18: 168 (1881). Type: Mozambique, near Sena, 8 April 1860, J. Kirk 34 (K, holo.!)

A. lugardiana Baker in Bull. Misc. Inform., Kew 1901: 135 (1901). Type: Botswana, Botletle River, near Sibetuane's Drift, 30 June 1897, E.J. Lugard 2 (K, holo.!)

A. platyphylla Baker in Trans. Linn. Soc. London, Bot. 1: 264 (1878). Type: Angola, Pungo Andongo, 1879, F. Welwitsch 3722 (K, lecto.; BM, $\mathrm{G}$, LISU, isolecto.)

Diagnostic characters: This aloe sometimes occurs as solitary plants, but usually forms dense groups. Leaves are sometimes more distinctly spotted on the lower surface than the upper surface (Figure 8). Inflorescences are 1.0-1.6(-2.0) m high and branched from above the middle. Flowers are $30-35 \mathrm{~mm}$ long and dull red, with a dull whitish border on the outer perianth segments. Distribution: Angola (Bengo, Cuando-Cubango, Cuanza Sul, Cunene, Huambo, Huíla, Malange, Moxico), Botswana, western Mozambique, 
Malawi, northern Namibia, South Africa (NorthWest), Zambia, Zimbabwe.

Habitat: Variety of veld types and soil, mostly grassland and thickets on dry hills. Also abundant in open Colophospermum mopane woodland.

Aloe braamvanwykii Gideon F. Sm. \& Figueiredo spec. nov.

Type: South Africa: North-West Province, on the farm Leeuwfontein 185, near Baskop alongside the tarred Leeufontein road, about $4 \mathrm{~km}$ from Wolmaransstad, 5 January 2012, Abrie Steyn, Gideon F. Smith \& Estrela Figueiredo 1 (PRE, holo.)

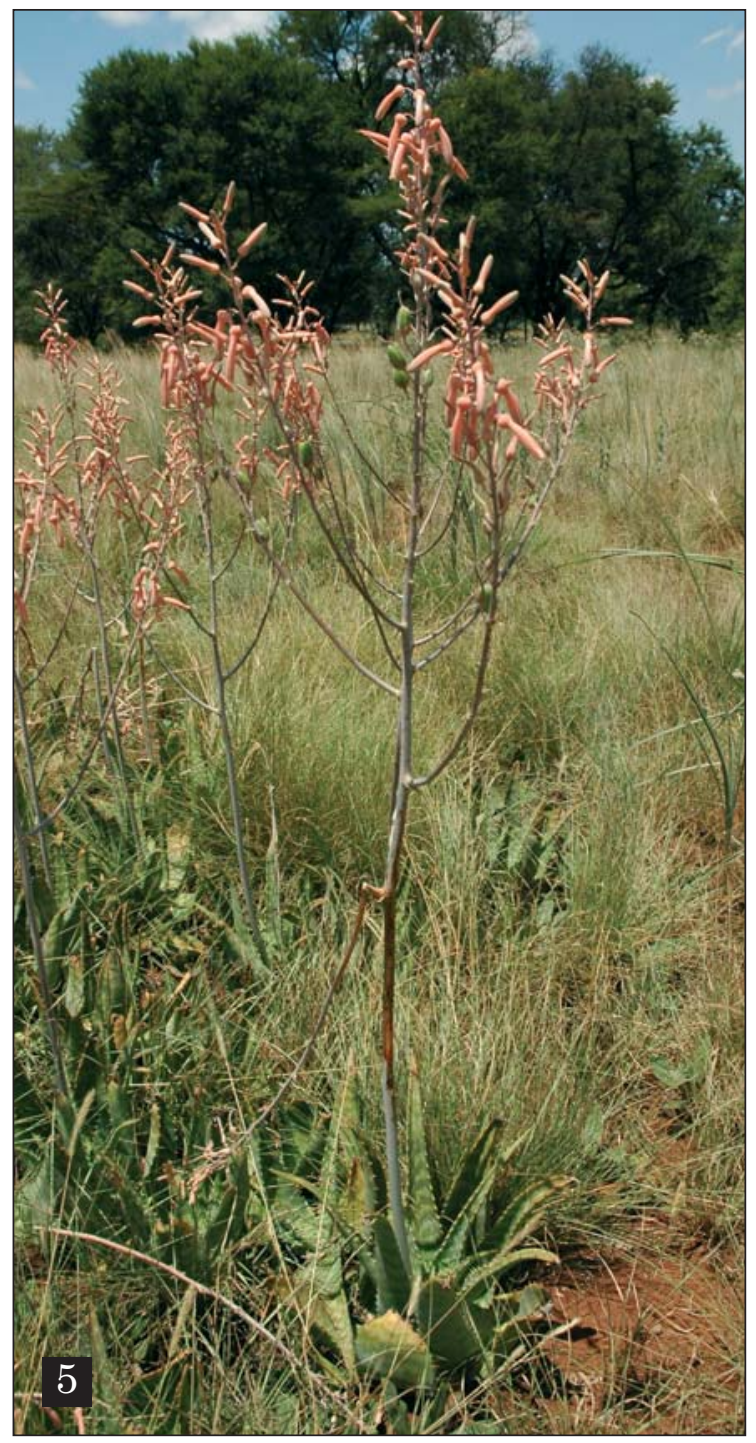

Small to medium-sized, herbaceous, slowgrowing, succulent, perennial, maculate aloe, total height excluding inflorescence $0.17-0.28 \mathrm{~m}$, usually clumped, 5-70 heads, sometimes solitary, a single head up to (17-)22 $\mathrm{cm}$ in diameter. Roots cylindrical, $5 \mathrm{~mm}$ in diameter. Stems absent or, if rarely present, very short. Leaves few, $12-15$, rosulate, rigidly spreading to erect, persistent when dry, dull mid-green, upper surface slightly concave, hardly canaliculate, with numerous scattered white spots throughout, spots arranged in irregular transverse bands; lower surface convex, white spots more distinctly arranged in transverse bands, sometimes confluent yielding milky

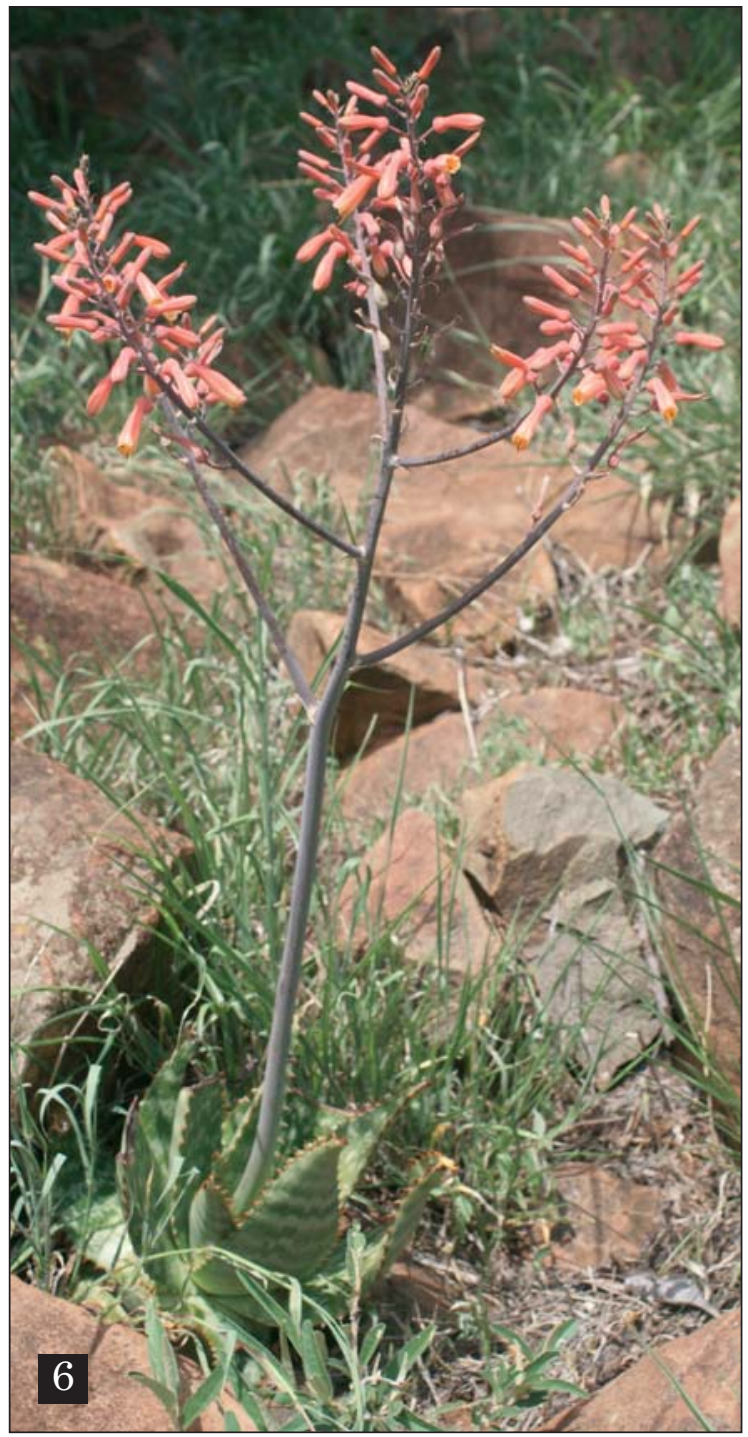

Figure 5. A. transvaalensis has dull milky green leaves with obscurely spotted lower surfaces and fleshpink flowers. Photo: Ronell R. Klopper. Figure 6. A. braamvanwykii in habitat near Wolmaransstad, North-West Province, South Africa. Photo: Gideon F. Smith. 

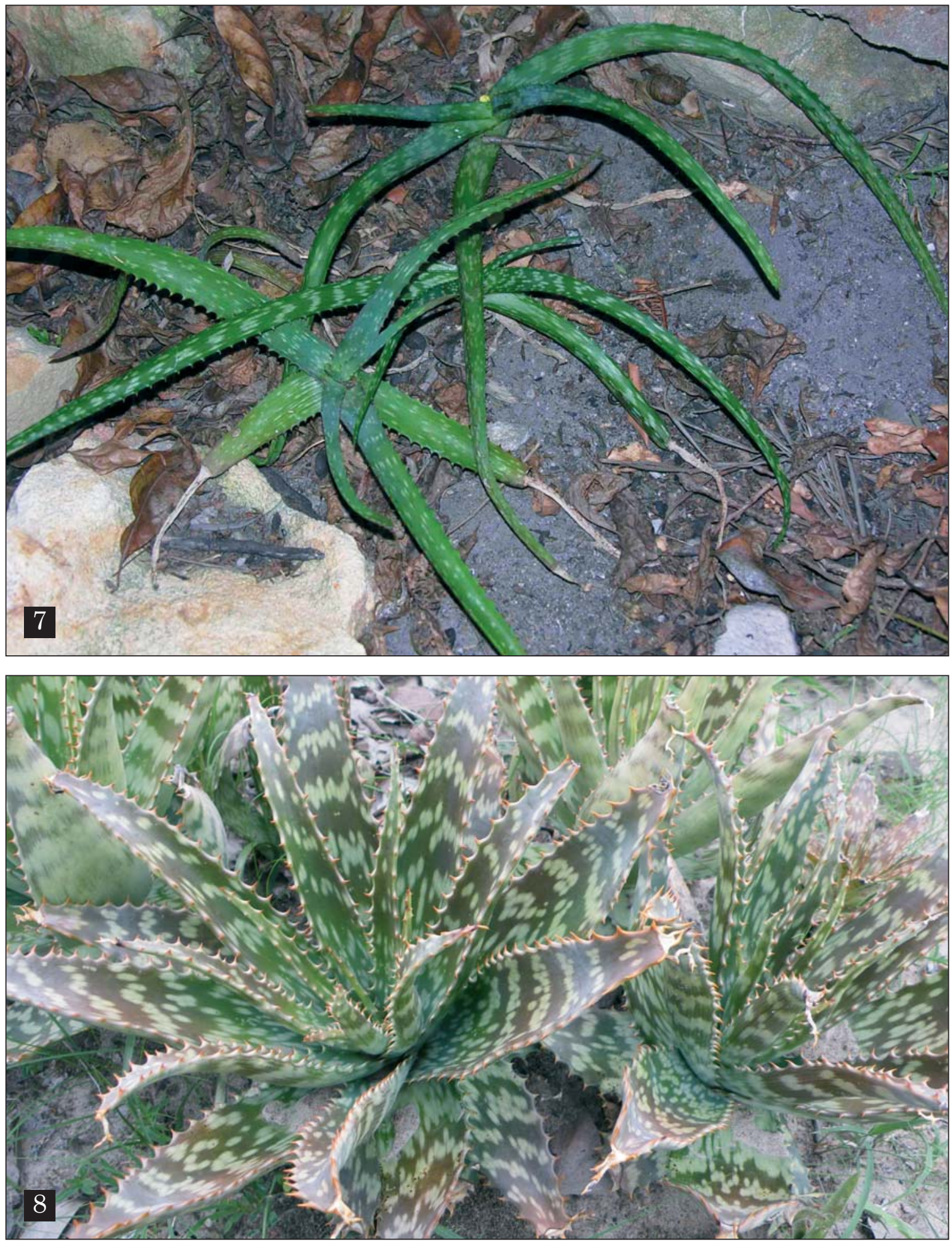

Figure 7. A. vandermerwei has very long, narrow, curved and almost snake-like leaves. Photo: Philip Nel. Figure 8. Rosettes of A. zebrina showing the distinctly marked upper and lower surfaces. Photo: Ernst J. van Jaarsveld. 

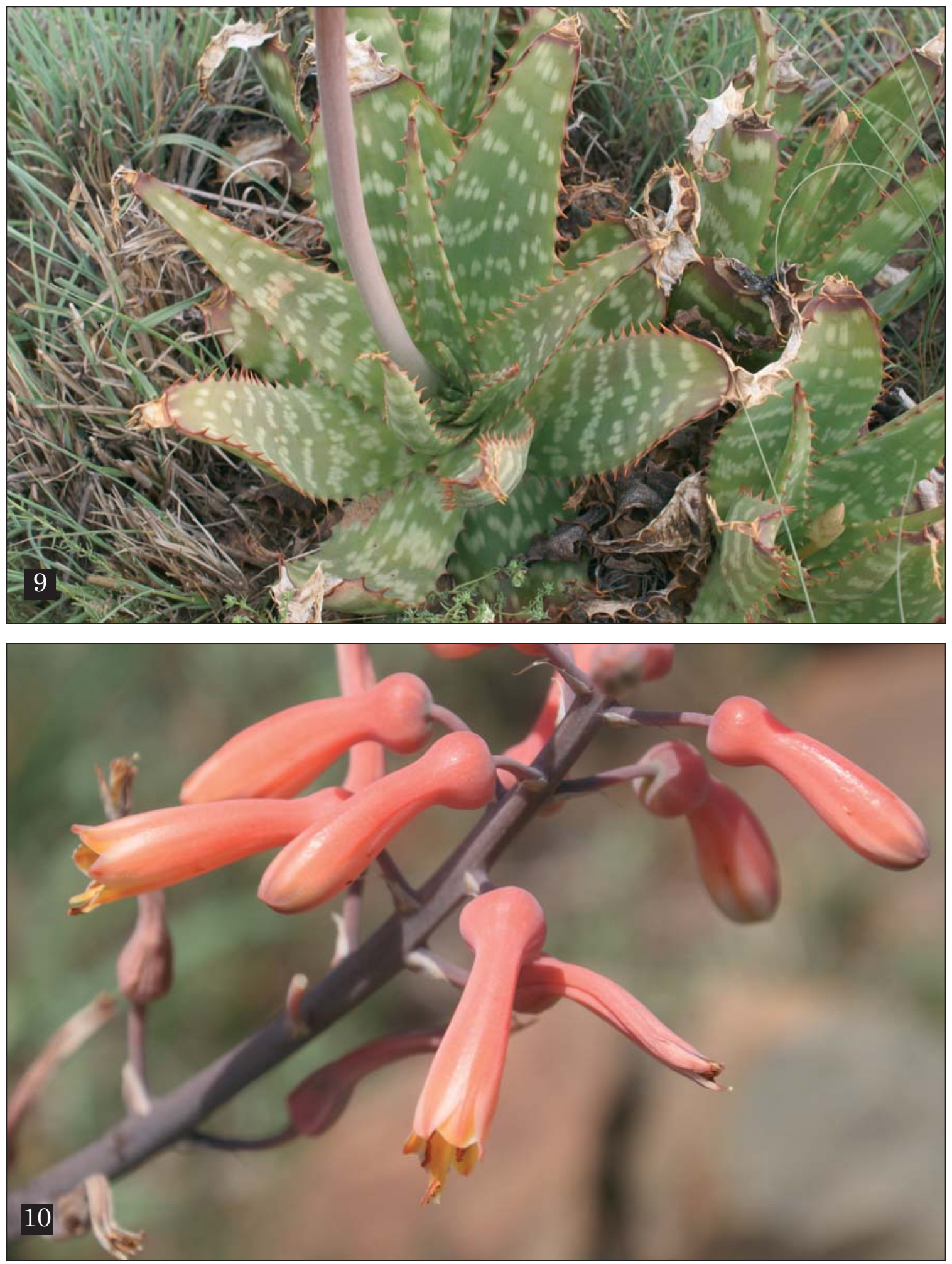

Figure 9. Close-up of a rosette of A. braamvanwykii. Figure 10. Close-up of the bright red flowers of A. braamvanwykii. Photos: Gideon F. Smith. 
green surface, texture smooth, linear-attenuate, tapering to apex, $17-26 \mathrm{~cm}$ long, $3.5-5.5 \mathrm{~cm}$ broad at base, basally sheathing; margins very thin, brown, with triangular marginal teeth, green with light brown tips, $\pm 4 \mathrm{~mm}$ long, same length throughout, evenly spaced at 10-13 mm apart; exudate pale yellowish, drying purple. Inflorescence $1-3$, successively, $0.65-0.75 \mathrm{~m}$ tall, far exceeding the height of rosette, central raceme longest, 5-7branched from above middle, branches arcuateerect. Peduncle 270-420 $\mathrm{mm}$ long, 8-14 mm broad at base, basally plano-convex, cylindrical above, light greenish brown with a white, powdery bloom; not sterile bracteate; bracts subtending racemes narrowly triangular, $15-65 \mathrm{~mm}$ long, 6$8 \mathrm{~mm}$ broad at the base, straw-coloured to light brown, papery, rarely fleshy, many nerved. Racemes cylindrical, 14-17 cm long, 3-5 cm wide; buds erect to suberect, flowers horizontal to drooping when mature. Floral bracts narrowly triangular, long attenuate, amplexicaul around pedicel, 5-9 mm long, 4-5 mm wide, straw-coloured, papery, 3-4 nerved. Pedicels 10-12 mm long, pinkish brown. Flowers: actinomorphic to slightly zygomorphic, unscented, nectariferous; perianth greenish tipped in buds, somewhat bicoloured when mature, light pink to mainly orange-red to bright red with whitish to yellowish longitudinal stripes, tip extremity purplish-brown or whitish, lightly pruinose, $20-25 \mathrm{~mm}$ long, flattened at base, $\pm 6 \mathrm{~mm}$ across ovary, distinctly narrowed above ovary to $\pm 3 \mathrm{~mm}$ to form globose basal swelling, enlarging to 6-7 $\mathrm{mm}$ towards throat and wide open mouth, tubular-cymbiform; outer segments larger than inner segments, lorate, free for $\pm 7 \mathrm{~mm}$, free portion centrally pinkish red, borders white or light yellowish, acute, segment margins straight, tips slightly recurved; inner segments narrower than outer, with white or yellowish border and more obtuse apex, free for upper $2 / 3$ of their length; stamens with cylindrically threadlike to very slightly flattened, light yellow filaments, 25-28 mm long, all 6 of \pm equal length, exserted for 2-5 mm; anthers small, 1-2 mm long, dark brown, versatile; ovary $5-6 \mathrm{~mm}$ long, $3 \mathrm{~mm}$ in diameter, light green; style as long as or slightly longer than stamens, minutely capitate, with small stigma, exserted 1-2 mm. Fruit an erect, bright green, cylindrical, trilocular capsule, 17-22 mm long, 9-11 $\mathrm{mm}$ in diameter, apically truncate, dry remains of tepals shed from around fruit early on, dehiscing loculicidally, chartaceous when dry, apically valves sigmoidally curved outwards. Seeds dark greyish brown, angled, laterally compressed, $2.5-3.0 \mathrm{~mm}$ long, with up to $1 \mathrm{~mm}$ wide off-white wing stretching around periphery of seed. Chromosome number unknown.
Flowering time: December to February.

Diagnostic characters: This species is geographically isolated from other members of the A. zebrina-complex. Hitherto its existence has not been reflected by range maps for the complex as a whole, nor for any of the constituent species. It is probably most closely related to $A$. transvaalensis. Plants are long-lived and typically form dense clumps of up to 70 heads (Figures $6 \& 9$ ). Not only are its flowers the smallest (20-25 $\mathrm{mm}$ long) among South African members of the A. zebrinacomplex, but they are also characteristic in being an unusual intense red (Figure 10). Also diagnostic are the general small stature of the plants and shorter inflorescences $(0.65-0.75 \mathrm{~m})$. An outstanding feature is its concentrated and relatively early (December to February) flowering period. Despite being subjected to probably the coldest winter temperatures, this species is the earliest of the local members of the complex to flower. Plants are consistently in bloom on Christmas Day (25 December), but flowering is brief and does usually not extend beyond the end of February.

Distribution: A. braamvanwykii is only known from the Wolmaransstad, Schweizer-Reneke, Delareyville and Stella area, North-West, South Africa. The species has a very restricted distribution range with all known localities less than $50-100 \mathrm{~km}$ apart. Most of the natural vegetation surrounding the known range of the species has been destroyed for cultivation. However, the reasons for the species' restricted distribution are not obvious. It is absent from fairly extensive areas of seemingly similar natural habitat (see below) towards Klerksdorp and Leeudoringstad.

Habitat: Plants are associated with relict stands of Klerksdorp Thornveld (Mucina \& Rutherford, 2006), a vegetation type characterized by unevenly scattered Acacia karroo-dominated tree stands in a grassland matrix. Plants mainly grow in full sun in open grassy areas among woody vegetation. Other commonly associated trees and shrubs include Acacia hebeclada subsp. hebeclada, Acacia robusta subsp. robusta, Asparagus laricinus, Diospyros lycioides subsp. lycioides, Ehretia alba, Grewia flava, Searsia ciliata and Tarchonanthus camphoratus. Prominent grasses include Anthophora pubescens, Cymbopogon pospischilii, Digitaria eriantha, Eragrostis superba and Themeda triandra. Plants are typically associated with Lippia scaberrima, a highly aromatic perennial forb. Aloe braamvanwykii prefers red sandy loam (often with small stone aggregates) derived from rocks of the Ventersdorp Supergroup (Johnson et al., 2006), but occasionally can also be found on more clay-rich soils. Average annual rainfall is about $375 \mathrm{~mm}$ and falls 
mainly in summer. Winter nights are cold and subzero temperatures with frost are common.

Eponymy: Aloe braamvanwykii is named after Prof. Braam van Wyk, from the University of Pretoria, who called our attention to this plant. If the epithet 'braamii' (excluding his surname therefore) was chosen, it could be confused with $A$. broomii Schönland, especially if written by hand. Moreover, several contemporary South African botanists go by the surname 'Van Wyk', which influenced our decision to unambiguously call the species A. braamvanwykii. Braam co-authored the book on aloes in southern Africa (Smith \& Van Wyk, 2008) with one of us (GFS).

Further specimens: South Africa, Northwest. -2624: About $12 \mathrm{~km}$ north of Stella, on road to Papiesvlakte, (-BD), 3 March 1998, L. Smook 10103 (PRE). 2625: Baberspan Nature Reserve, (-DA), 12 March 1973, N. Zambatis 75 (PRE). 2725: Schweizer-Reneke district, $(-\mathrm{AB}), 16$ February 1959, E. Werdermann \& H.D. Oberdieck 2242 (PRE).

\section{Acknowledgements}

Permission granted by Mr Pieter and Mrs Jess Steyn to conduct fieldwork on their farm is gratefully acknowledged. Mr Abrie Steyn accompanied us during the fieldwork. Prof. A.E. (Braam) van Wyk kindly shared his notes on $A$. braamvanwykii with us. Various photographers generously provided images. An anonymous referee is thanked for suggesting improvements to the manuscript.

\section{References}

Carter, S., Lavranos, J.J., Newton, L.E. \& Walker, C.C. (2011). Aloes: the definitive guide. Royal Botanic Gardens, Kew, London.

Glen, H.F. \& HARDY, D.S. (2000). Aloaceae (First part): Aloe. In GERMishuIZEN, G. (ed.), Flora of southern Africa, Vol. 5, Part 1, Fascicle 1: 1167. National Botanical Institute, South Africa.

Glen, H.F., Smith, G.F. \& Hardy, D.S. (1995). Typification of Aloe species described by H.B. Groenwald. Bothalia 25: 97-99.

Groenewald, H.B. (1937a). 'n Nuwe Aloe van die Laeveld. Tydskr. Wetensk. Kuns 15: 126-128.

Groenewald, H.B. (1937b). 'n Nuwe Aloe uit Lourenço Marques. Tydskr. Wetensk. Kuns 16: $13-15$.

Hargreaves, B.J. (1990). The succulents of Botswana. National Museum, Monuments and Art Gallery, [Gaborone], Botswana.

Johnson, M.R., Anhaeusser, C.R. \& Thomas, R.J. (eds) (2006). The geology of South Africa. Geological Society of South Africa, Johannesburg/Council for Geoscience, Pretoria.

Mucina, L. \& Rutherford, M.C. (eds.) (2006). The vegetation of South Africa, Lesotho and Swaziland. Strelitzia 19. South African National Biodiversity Institute, Pretoria.

ReYnolds, G.W. (1950). The Aloes of South Africa. The Aloes of South Africa Book Fund, Johannesburg.

Sмith, G.F. \& Van Wyk, A.E. [Braam]. (2008). Aloes of southern Africa. Struik Nature, Cape Town.

VAn WyK, B-E. \& Sмith, G.[F.] (1996). Guide to the aloes of South Africa. Briza Publications, Pretoria. 